\title{
Efeito de diferentes níveis de suplementação concentrada sobre o desempenho reprodutivo de vacas leiteiras mantidas a pasto ${ }^{1}$
}

\author{
Effect of different levels of concentrate supplement on the \\ reproductive performance of dairy cows maintained to pasture
}

\author{
Rafael Salmazo ${ }^{2}$, Ivone Yurika Mizubuti ${ }^{3 *}$, Fernanda Barros Moreira ${ }^{4}$, \\ Marco Antonio da Rocha ${ }^{4}$, Edson Luis de Azambuja Ribeiro ${ }^{3}$, \\ Marcelo Marcondes Seneda ${ }^{4}$, Paulo Tadatoshi Hiroki ${ }^{5}$, Cristina Célia Krawulski ${ }^{6}$, \\ André Gomiro Rigo ${ }^{7}$, Rafael Vasconcelos Schroeder ${ }^{7}$
}

\begin{abstract}
Resumo
Este experimento teve como objetivo avaliar os efeitos de diferentes níveis de suplementação concentrada nos períodos pré-parto e pós-parto, sobre o desempenho reprodutivo de vacas leiteiras mantidas a pasto. Utilizaram-se 54 vacas em delineamento experimental inteiramente casualizado, distribuídas em esquema fatorial $3 \times 2$, sendo 3 tratamentos, 2 períodos e 9 repetições por tratamento. Os tratamentos compreenderam diferentes níveis de suplementação de concentrado (em base seca) nos períodos pré-parto (PRE): PRE1 $=0 \%$ do PV; PRE2 $=0,5 \%$ do PV; PRE3 $=1,0 \%$ do PV; e no pós-parto (POS): $P O S 1=1 \mathrm{~kg}$ para cada $2,5 \mathrm{~kg}$ de leite produzido; $\mathrm{POS} 2=1 \mathrm{~kg}$ para cada $2,0 \mathrm{~kg}$ de leite produzido e POS3 $=1 \mathrm{~kg}$ para cada $1,5 \mathrm{~kg}$ de leite produzido, acima de $6 \mathrm{~kg}$ de leite por dia. Os animais foram submetidos à avaliação ultrasonográfica e de escore corporal a cada 7 dias. No período avaliado, 20 animais apresentaram cio e 13 animais conceberam. A média de intervalo entre parto-primeiro cio foi de 53,13 dias $(\mathrm{P}<0,05)$. Com o aumento do nível de suplementação pós-parto, aumentou-se o numero de animais que entraram em cio, sendo de 2, 4 e 7 animais para os tratamentos 1,2 e 3, respectivamente. $\mathrm{O}$ único tratamento em que todos os animais conceberam, foi o tratamento PRE3 e POS3. Houve maior número de concepções entre os animais que receberam tratamento 2 e 3 , tanto no pré quanto no pósparto, em relação aos que receberam o tratamento $1(\mathrm{P}<0,05)$. $\mathrm{O}$ aumento do nível de suplementação nos tratamentos pré e pós-parto, provocaram diminuição linear do número de semanas para início de atividade folicular (IAF) e início de crescimento folicular (ICF). Houve correlação negativa entre o IAF $(-0,34)$, ICF $(-0,31)$ e escore corporal ao parto. Conclui-se que, o aumento no nível de suplementação tanto no pré quanto no pós-parto, aumenta a quantidade de animais que manifestam cio e concebem nos primeiros 120 dias pós-parto, assim como na manutenção da gestação. Ocorre diminuição linear no tempo para IAF e ICF à medida que se aumenta o nível de suplementação no pré e pós-parto, sendo estes correlacionados com o escore corporal ao parto.
\end{abstract}

Palavras-chave: Alimentação, balanço energético, bovino, pré-parto, pós-parto

Parte da dissertação de mestrado do primeiro autor

Médico Veterinário da Secretaria de Agricultura e Abastecimento- Paraná

3 Prof. Dr. do Departamento de Zootecnia e do Programa de Pós-graduação em Ciência Animal da Universidade Estadual de Londrina (UEL). Bolsista de Produtividade do CNPq. E-mail: mizubuti@uel.br

4 Prof. Dr. do Departamento de Zootecnia e do Programa de Pós-graduação em Ciência Animal da Universidade Estadual de Londrina (UEL).

5 Médico Veterinário. Emater, Londrina, PR

6 Agrônoma. Emater, Jaguapitã, PR.

7 Médicos Veterinários.

* Autor para correspondência 


\begin{abstract}
This experiment was carried out with the objective of evaluating the effects of different levels of concentrate supplement in the pre and post-parturition periods on the reproductive performance of dairy cow. 54 cows in a completely randomized design, distributed in $3 \times 2$ factorial arrangement, being 3 treatments, 2 periods and 9 repetitions for treatment, were used. The treatments with different levels of concentrate supplement (in dry basis) in the pre-parturition periods (PRE), were: PRE1 $=0 \%$ of body weight $(\mathrm{BW})$; PRE2 $=0.5 \%$ of $\mathrm{BW}$; PRE3 $=1.0 \%$ of BW; and in the post-parturition (POS), were: POS $1=1 \mathrm{~kg}$ for each $2.5 \mathrm{~kg}$ of produced milk; $\mathrm{POS} 2=1 \mathrm{~kg}$ for each $2.0 \mathrm{~kg}$ of produced milk and POS 3 $=1 \mathrm{~kg}$ for each $1.5 \mathrm{~kg}$ of produced milk, above $6 \mathrm{~kg}$ of milk a day. The ultrasonography evaluation and body score were evaluated every seven days. 20 animals presented estrus and 13 animals became pregnant in the evaluated period. The average interval between parturition-first estrus was of 53.13 days, varying from 19 to 105 days. The amount of animals that entered in the estrus in the 120 days of evaluation increased with the post parturition supplementation level, being of 2, 4 and 7 animals for the treatments 1, 2 and 3, respectively. For the conception, the only treatment in that all the animals became pregnant, was the treatment PRE3 and POS3. There was higher number of conceptions among the animals that received treatment 2 and 3 , so much in the pre as in the post-parturition, in relation to animals that received the treatment 1 . The increasing supplement level in the treatments pre and postparturition caused lineal decrease of the number of weeks for beginning of follicular activity (BFA) and beginning of follicular growth (BFG). There was negative correlation among BFA (-0.34), BFG (-0.31) and body score to the parturition. It can be concluded that the increasing supplement level in the pre and post-parturition treatments, increases the amount of animals that manifest estrus and become pregnant in the first 120 days post-parturition, as well as, in the pregnant maintenance. Lineal decrease occurred in the time for BFA and BFG as increases the supplement level in the pre and post- parturition, being these correlated with the body score to the parturition.
\end{abstract}

Key words: Feeding, energy balance, bovine, pre-parturition, post-parturition

\section{Introdução}

Ao longo dos anos pode-se observar que houve aumentonaproduçãodeleite, devidoaomelhoramento genético, mas houve também diminuição na taxa de concepção, nos rebanhos leiteiros, sendo que essa tendência pode ser evidenciada em todo o mundo. No entanto, a diminuição da fertilidade não está diretamente ligada ao aumento na produção de leite dos rebanhos, mas sim ao fato de que os avanços na genética não foram acompanhados de melhorias no manejo sanitário, reprodutivo e principalmente nutricional (LUCY, 2001).

No Brasil, uma das principais razões do baixo desempenho produtivo e reprodutivo de bovinos é a baixa disponibilidade de alimentos e o inadequado manejo nutricional do rebanho (SANTOS; AMSTALDEN, 1998). Dentre os aspectos nutricionais, o que mais interfere no desempenho reprodutivo de vacas leiteiras, no pós-parto imediato, é o balanço energético negativo, porém a proteína, as vitaminas e os minerais também têm grande importância na reprodução.

Lucy, Staples e Michel (1991) descreveram que o balanço energético negativo e a perda de peso inibem o crescimento e o desenvolvimento. Com o aumento no desempenho animal, há um acréscimo nas exigências nutricionais para síntese de leite e de tecidos, que podem afetar as funções reprodutivas (SANTOS; AMSTALDEN, 1998). Essas exigências são maximizadas nas vacas em início de lactação, pois não consegue consumir quantidades suficientes de energia para atender seus requerimentos de manutenção e produção, o que acarreta um inevitável balanço energético negativo (BUTLER; SMITH, 1989). Durante o início da lactação, os mecanismos de partição de nutrientes dão prioridade à produção de leite, em detrimento das funções reprodutivas. Segundo Roche, Mackey e Diskin (2000), o manejo nutricional de vacas 
leiteiras no período de transição, ou seja, entre três semanas antes e três depois do parto, tem efeito significativo na eficiência reprodutiva.

Devido à importância que assume a reprodução na rentabilidade e eficiência dos rebanhos leiteiros, e devido a resultados discordantes na literatura, mais investigações são necessárias com animais de diferentes status genético e condições de manejo.

O objetivo desse estudo foi avaliar o efeito de diferentes níveis de suplementação de concentrado nos períodos pré e pós-parto dos animais sobre o desempenho reprodutivo no pós-parto, nos parâmetros de tempo para primeiro cio, taxa de concepção, tempo para concepção e desenvolvimento folicular de vacas leiteiras.

\section{Material e Métodos}

Este experimento foi realizado em propriedade particular, assistido pelo Projeto Vitória EmaterParaná, e localizada no município de Jaguapitã, Estado do Paraná.

O delineamento experimental foi inteiramente casualizado, distribuídos em esquema fatorial 3 x 2, sendo 3 tratamentos (níveis de concentrado), 2 períodos (pré e pós-parto) e 9 repetições por tratamento, totalizando 54 vacas mestiças ( 27 por período). A distribuição dos animais nos tratamentos foi casualizada, sendo que os animais dos tratamentos no período pré-parto, foram divididos igualmente entre os tratamentos no período pós-parto.

Os animais entraram no experimento, 30 dias antes da provável data do parto (PDP), para compor o período pré-parto (PRE) e receberam a suplementação correspondente a esse período. Após o parto, os animais foram redistribuídos para compor o período pós-parto (POS) e receberam as respectivas suplementações durante 60 dias e foram avaliados nos 120 dias subseqüentes ao parto.

Os tratamentos compreenderam a administração de três níveis de suplementação com concentrado, em base seca, no período pré-parto (PRE) e três no período pós-parto (POS), sendo:

PRE1 $=0 \%$ do peso vivo (PV) (controle);

PRE2=0,5\% do PV (nível médio de concentrado);

PRE3= 1,0\% do PV (nível alto de concentrado);

POS1 $=1 \mathrm{~kg}$ para cada $2,5 \mathrm{~kg}$ de leite produzidos acima de $6 \mathrm{~kg}$ (controle);

POS2 $=1 \mathrm{~kg}$ para cada $2,0 \mathrm{~kg}$ de leite produzidos acima de $6 \mathrm{~kg}$ (nível médio de concentrado);

POS3 $=1 \mathrm{~kg}$ para cada $1,5 \mathrm{~kg}$ de leite produzidos acima de $6 \mathrm{~kg}$ (nível alto de concentrado).

O tratamento POS1, foi representado pela quantidade de concentrado suficiente para suprir a produção de leite do animal, sendo esta de $1 \mathrm{~kg}$ de concentrado para cada $2,5 \mathrm{~kg}$ de leite produzido (NATIONAL RESEARCH COUNCIL, 2001). O nível médio e alto foi calculado visando fornecer, respectivamente, $25 \%$ e $50 \%$ mais energia do que o grupo controle, portanto, $1 \mathrm{~kg}$ de concentrado para cada $2,0 \mathrm{~kg}$ e $1,5 \mathrm{~kg}$ de leite produzido, respectivamente.

O concentrado foi fornecido pela Cooperativa Agroindutrial Integrada e continha 10,17\% de umidade e a seguinte composição química (\%), em base seca: extrato etéreo $(\mathrm{EE})=2,65$; fibra bruta $(\mathrm{FB})=10,03$; cinzas $=7,97$; cálcio $(\mathrm{Ca})=1,14$; fósforo $(\mathrm{P})=0,77$; proteína bruta $(\mathrm{PB})=20,83 \mathrm{e}$ $75 \%$ de nutrientes digestíveis totais (NDT).

A suplementação, conforme o tratamento, foi fornecida pela manhã para os animais no pré-parto, e pela manhã e à tarde no pós-parto (no momento da ordenha).

O período experimental foi de agosto de 2004 a julho de 2005, onde as parições se concentraram de setembro de 2004 a abril de 2005 . Os animais foram mantidos em pastejo de grama estrela roxa (Cynodon Nlemfuensis) durante todo o período experimental, tendo à disposição sal e água ad libitum, em cochos de fácil acesso. O pasto era dividido em 10 piquetes 
de aproximadamente 0,8 ha cada, sendo o período de ocupação médio de 3 dias.

Para avaliação da pastagem, que era predominantemente da gramínea estrela roxa (Cynodon Nlemfuensis), foram coletadas 9 amostras de $0,25 \mathrm{~m}^{2}$ a cada 28 dias, conforme técnica descrita por Houlderbaun e Sollennberg (1992). Estas amostras foram separadas em duas porções: planta inteira e folha + colmo + material morto. As duas porções foram secas em estufa a $55 \pm 5^{\circ} \mathrm{C}$ por $72 \mathrm{~h}$ horas e moídas em moinho tipo faca com peneira de $1 \mathrm{~mm}$ de crivo, para posteriores análises. Desta forma, foram determinadas durante o período, a porcentagem e quantidade média, em toneladas, de folha, colmo, e material morto, com a finalidade de avaliar a quantidade e qualidade da pastagem disponível para os animais.

As análises químicas foram realizadas no Laboratório de Nutrição Animal da Universidade Estadual de Londrina. Foram determinados os teores de matéria seca e proteína bruta da forragem (Tabela 1) (ASSOCIATION OF OFFICIALANALYTICAL CHEMISTS, 1990).

Tabela 1. Quantidade média de colmo, matéria morta e folha (em toneladas e \%) por hectare, e composição da pastagem em matéria seca (MS) e proteína bruta (PB).

\begin{tabular}{|c|c|c|c|c|}
\hline \multirow{2}{*}{ Parte da planta } & \multicolumn{2}{|c|}{ Quantidade média/ ha } & \multirow{2}{*}{$\begin{array}{c}\text { MS TOTAL } \\
(\%)\end{array}$} & \multirow{2}{*}{$\begin{array}{c}\text { PB } \\
(\% \text { na } M S)\end{array}$} \\
\hline & ton & $\%$ & & \\
\hline Colmo & 13,37 & 47,76 & 56,28 & 4,82 \\
\hline Matéria morta & 6,93 & 24,86 & 67,22 & 3,48 \\
\hline Folha & 7,70 & 27,50 & 58,28 & 9,38 \\
\hline
\end{tabular}

Foram realizadas as pesagens do leite e a avaliação da condição corporal dos animais semanalmente, sempre no mesmo dia da semana. As determinações do escore corporal foram realizadas, utilizando-se escala de 1 a 5 pontos (1-muito magra até 5-obesa, segundo EDMONSON et al., 1989). A pesagem do leite foi realizada 2 vezes ao dia no momento da ordenha, sendo considerado como total produzido, a soma das duas pesagens.

No período pós-parto, os animais foram submetidos semanalmente a ultra-sonografia e avaliação da condição corporal.

A avaliação reprodutiva baseou-se na observação feita pelo proprietário com relação ao aparecimento de cio e avaliações com ultra-som a cada sete dias, segundo recomendações de Pierson e Ginther (1984), onde eram observadas a presença de conteúdo uterino e as estruturas ovarianas.
O aparecimento de cio foi confirmado com a presença da imagem de corpo lúteo no ultra-som. A dinâmica ovariana no período pós-parto foi avaliada pela mensuração do diâmetro dos folículos no ultrasom e do período de aparecimento de tais estruturas. Dessa forma, obtiveram-se os dados de início de atividade folicular (IAF), representada pelos folículos maiores que $5 \mathrm{~mm}$, e início de crescimento folicular (ICF), em que os folículos apresentavam diâmetro acima de $8 \mathrm{~mm}$. Tanto o ICF quanto o IAF, estão representados como semanas pós-parto (SPP) por essas avaliações terem sido realizadas a cada 7 dias.

A avaliação da condição corporal dos animais foi realizada semanalmente, sempre no mesmo dia utilizando-se escala de 1 a 5 pontos (1 muito magra, até 5 obesa segundo EDMONSON et al., 1989).

Os dados foram analisados utilizando-se o programa de análises estatísticas SAEG, conforme o seguinte modelo matemático: 


$$
\mathrm{Y}_{\mathrm{ijk} \mathrm{k}=} \mu+\mathrm{TE}_{\mathrm{i}}+\mathrm{TO}_{\mathrm{j}}+\mathrm{N}_{\mathrm{k}}+(\mathrm{TETO})_{\mathrm{ij}}+\mathrm{E}_{\mathrm{ijkl}}
$$

Onde:

$\mathrm{Y}_{\mathrm{ijk}}=$ Observação do Início de Atividade Folicular e Início de Crescimento Folicular, nos tratamentos dos períodos pré (i) e pós parto (j) e interação dos tratamentos pré e pos parto (ij) e número de parto $(\mathrm{k})$. $\mu=$ media geral $\mathrm{TE}_{\mathrm{i}=}$ efeito do tratamento no pré - parto, sendo $\mathrm{i}=1$, 2 e 3 ;

$\mathrm{TO}_{\mathrm{j}=}$ efeito do tratamento no pos- parto, sendo $\mathrm{j}=1$, 2 e 3 ;

$\mathrm{N}_{\mathrm{k}=}$ efeito do numero de parto, sendo $\mathrm{k}=1,2,3,4 \mathrm{e}$ 5 ;

$(\text { TETO })_{\mathrm{ij}=}$ efeito da interação dos tratamento no préparto (i) com o pós-parto (j); $\mathrm{E}_{\mathrm{ijk} \mathrm{l}=}$ Erro experimental associado a cada McNamara et al. (2003). observação.

\section{Resultados e Discussão}

Dos 27 animais avaliados, foram constatados quatro animais com cistos ovarianos, dois com morte embrionária precoce e seis com infecção uterina, não sendo observado influência de tratamentos. Do total de animais avaliados, 20 apresentaram cio e 13 conceberam no período avaliado (Tabela 2 ).

A média de intervalo entre parto - primeiro cio foi de 53,13 dias, variando de 19 a 105 dias. Esse valor foi superior aos encontrados por Butler, Evereett e Coppock (1981), que observaram intervalo entre parto - primeiro cio de 36,6 dias. O intervalo partoconcepção apresentou valores médios de 66,07 dias, com máximo e mínimo de 88 e 30 dias, sendo estes valores inferior aos 105 dias encontrado por

Tabela 2. Influência do fornecimento de diferentes níveis de concentrado no período pré e pos parto sobre o desempenho reprodutivo das vacas

\begin{tabular}{|c|c|c|c|c|c|c|c|c|c|c|c|}
\hline TRAT & $\begin{array}{l}\text { PRE1 } \\
\text { POS1 }\end{array}$ & $\begin{array}{l}\text { PRE1 } \\
\text { POS2 }\end{array}$ & $\begin{array}{l}\text { PRE1 } \\
\text { POS3 }\end{array}$ & $\begin{array}{l}\text { PRE2 } \\
\text { POS1 }\end{array}$ & $\begin{array}{l}\text { PRE2 } \\
\text { POS2 }\end{array}$ & $\begin{array}{l}\text { PRE2 } \\
\text { POS3 }\end{array}$ & $\begin{array}{l}\text { PRE3 } \\
\text { POS1 }\end{array}$ & $\begin{array}{l}\text { PRE3 } \\
\text { POS2 }\end{array}$ & $\begin{array}{l}\text { PRE3 } \\
\text { POS3 }\end{array}$ & $\mathrm{EP} *$ & $\mathrm{P}^{*}$ \\
\hline NCIO & 1 & 2 & 2 & 1 & 3 & 3 & 2 & 3 & 3 & & \\
\hline NCON & - & 1 & 2 & - & 1 & 2 & 2 & 2 & 3 & & \\
\hline NTOTAL & 3 & 3 & 3 & 3 & 3 & 3 & 3 & 3 & 3 & & \\
\hline $\begin{array}{l}1^{\circ} \mathrm{CIO} \\
\text { (DPP) }\end{array}$ & 79 & 49 & 75 & 47 & 30,67 & 53 & 53,5 & 56 & 35 & 5,51 & 0,1511 \\
\hline $\begin{array}{c}\text { CONCEP } \\
\text { (DPP) }\end{array}$ & - & 53 & 75 & - & 98 & 63 & 53,5 & 70 & 50 & 5,35 & 0,3144 \\
\hline $\begin{array}{l}\text { IAF } \\
\text { (SPP) }\end{array}$ & 4,67 & 2,67 & 3,33 & 3,33 & 2,67 & 2,33 & 3 & 2,33 & 1,33 & 0,22 & 0,0397 \\
\hline $\begin{array}{l}\text { ICF } \\
\text { (SPP) }\end{array}$ & 3,67 & 2,67 & 3 & 3,33 & 2,67 & 2,33 & 3 & 2,33 & 1,33 & 0,17 & 0,0569 \\
\hline
\end{tabular}

NCIO - número de animais que entraram em cio; NCON - número de animais que conceberam até 120 dias; NTOTAL - número total de animais; DPP - dias pós-parto; SPP - semanas pós-parto; IAF - início de atividade folicular; ICF

- início de crescimento folicular.

*P - significância; $\mathrm{EP}=$ erro padrão. 
Com o aumento do nível de suplementação pós-parto, aumentou o número de animais que entraram no cio aos 120 dias de avaliação, sendo de 2, 4 e 7 animais para os tratamentos 1, 2 e 3, respectivamente. Com relação à concepção, o único tratamento em que todos os animais conceberam, foi o tratamento PRE3 e POS3. Observou-se também, maior número de concepções entre os animais que receberam o tratamento 2 e 3, tanto no pré (3 e 7 concepções, respectivamente) quanto no pós-parto (4 e 7 concepções, respectivamente), em relação aos que receberam o tratamento 1 (3 e 2 concepções, respectivamente no pré e pós-parto).

Não foi observada diferença estatística entre os tratamentos $(\mathrm{P}>0,05)$ em relação a dias pós-parto (DPP) para primeiro cio e concepção, embora numericamente, os animais do tratamento 1 , tanto do pré, quanto do pós-parto, tenham demorado mais dias para apresentarem o primeiro cio $(67,67$ e 59,83, respectivamente) em relação aos do tratamentos 2 (43,56 e 45,2, respectivamente) e 3 (48,18 e 54,33, respectivamente), sendo que essa diferença foi mais acentuada para o animal do tratamento pré-parto.

O fato de não ter havido diferença estatística para esses parâmetros deve-se, provavelmente, ao fato de que alguns animais não entraram em cio, nem conceberam no período avaliado de 120 dias pós-parto. Isso diminuiu o número de observações por tratamento, diminuindo o grau de liberdade, utilizado na análise estatística. Outro problema, é que essa variável depende da observação visual diária da pessoa responsável pelo manejo dos animais, estando dessa forma, sujeita, a erros. Thatcher e Wilcox (1972), relataram como sendo normal uma freqüência de $79 \%$ de cios silenciosos ou não observados. No entanto a avaliação da dinâmica folicular representada pelo início de atividade folicular (IAF) e início de crescimento folicular (ICF), realizada nesse experimento, podem anular esses problemas por serem avaliações baseadas em imagem ultrasonográfica, portanto mais objetivas.

Não houve diferenças estatísticas entre os tratamentos para número de partos, não sendo, portanto, a idade um fator que influencia este parâmetro.

Verificou-se também, que o aumento do nível de concentrado no pré e pós-parto, diminuiu a quantidade de animais que entraram no cio e não conceberam, sendo que do tratamento com maior nível de suplementação (PRE e POS), oito animais entraram em cio e apenas um não concebeu. Nos tratamentos 1 e 2 aproximadamente metade dos animais que entraram no cio, conceberam. Podese supor, portanto, que existe influência do nível nutricional e da condição corporal dos animais na concepção e/ou manutenção da gestação.

Brit (1994), relatou que o balanço energético negativo diminui a viabilidade do oócito contido no folículo ovulatório e a qualidade do corpo lúteo resultante da ovulação desse folículo. Da mesma forma, Gwazdauskas et al. (2000), coletaram oócitos durante o período de lactação, duas vezes por semana, por aspiração folicular, e demonstraram que o estágio de lactação e o nível de energia da dieta influenciaram a qualidade dos oócitos, pois animais recebendo dieta com menor nível energético, produziram oócitos com menor capacidade de fertilização. Segundo Snijders et al. (2000) a condição corporal pode afetar a qualidade dos oócitos, pois observaram que as fertilizações in vitro de oócitos provenientes de vacas de baixo escore corporal, tiveram baixa taxa de clivagem em relação àqueles animais com alto escore.

Para a maioria das espécies, o aumento do consumo de alimentos, leva a uma maior taxa de renovação hepática, aumentando a metabolização da progesterona e diminuindo seu nível circulante (LUCY, 2001). No entanto, para ovinos e bovinos, a circulação do ovário para o útero é feita pelo ducto ovariano sendo que a maior metabolização da progesterona não afeta a gestação (POPE; MAURER; STORMSHAK, 1982). Esse fato foi confirmado por Lozano, Abecia e Forcada (1998), ao observarem que ovelhas consumindo baixo teor 
de energia após a ovulação apresentaram altos teores de progesterona sanguíneos, porém menor concentração de progesterona uterina, quando comparadas com aquelas recebendo alto teor de energia após a ovulação.

A dispersão dos dados na análise da superfície de resposta para início de atividade folicular (IAF) e início de crescimento folicular (ICF) (Figuras 1 e 2), demonstra que o aumento dos níveis de suplementação concentrada no pré e pós-parto, provocou diminuição linear do número de semanas para o desenvolvimento folicular, e que o melhor desempenho foi observado na combinação PRE3POS3 (1,4 semanas) e o pior na PRE1-POS1 (4,3 semanas). Estes resultados diferem daqueles encontrados por Oxenreider e Wagner (1971), que relataram que a restrição no consumo de energia suficiente para causar redução no nível de glicose plasmática no pós-parto não causou efeito sobre o desenvolvimento de folículos maiores que $10 \mathrm{~mm}$ ou no intervalo da primeira ovulação.

Nota-se pelas Figuras 1 e 2, a influência dos tratamentos pré e pós-parto na melhora dos parâmetros reprodutivos avaliados, assumindo, portanto, um papel importante.

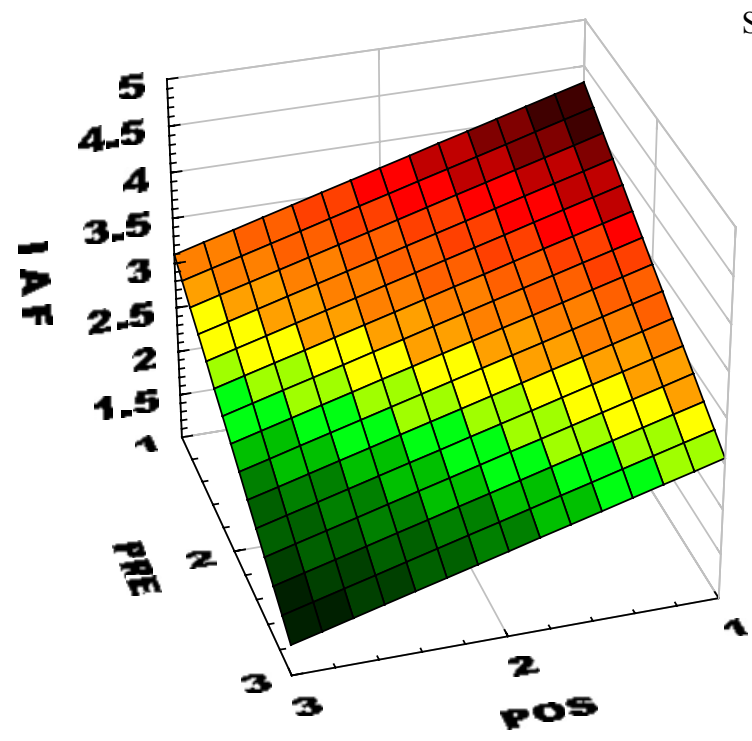

Figura 1. Influência do fornecimento de diferentes níveis de concentrado nos períodos PRÉ e PÓS parto sobre o início de atividade folicular (IAF).

Existe um mecanismo que explica a relação entre balanço energético e eficiência reprodutiva folicular, sobainfluência doLH(Hormônioluteinizante)eIGF1 (Insulin-like Growth Factor-1). Quando os pulsos de LH são deprimidos, decrescem as concentrações plasmáticas de IGF-1 que atuam de forma sinérgica com as gonadotrofinas (LH e FSH) para estimular o crescimento folicular. Além disso, os peptídeos IGF-1 e IGF-2 (Insulin-like Growth Factor-1 and 2), a insulina e o GH (growth hormone), exercem outras ações nos ovários dos bovinos, tais como, estimular a proliferação de células da granulosa; estimular a atividade da aromatase e a biosíntese de progesterona (SPICER; ENRIGHT, 1991). 


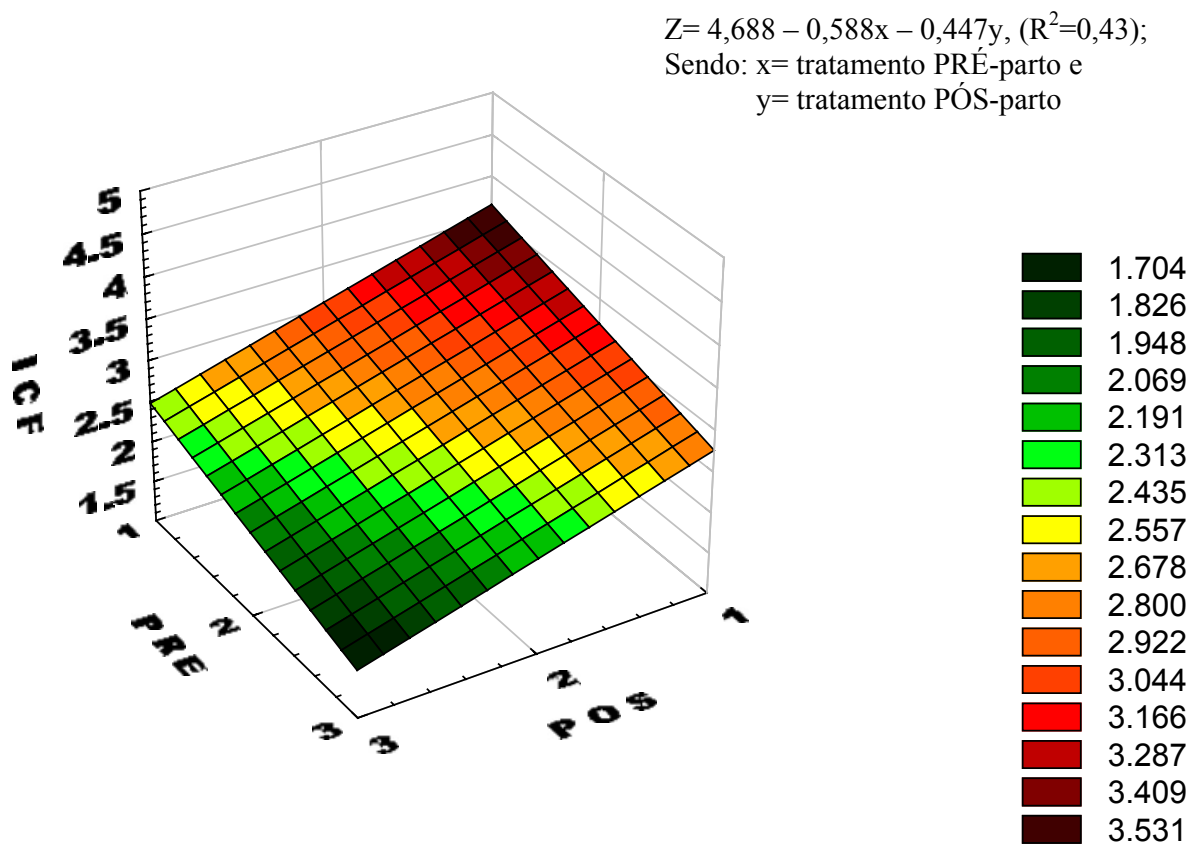

Figura 2. Influência do fornecimento de diferentes níveis de concentrado nos períodos PRÉ e PÓS parto sobre o início de crescimento folicular (ICF)

Animais recebendo dietas que promovem aumento de carboidratos plasmáticos tendem a ter o nível de insulina aumentado, e dentro dessa insulina, enquadra-se a IGF-1 e 2, que promovem acréscimo no desenvolvimento ovariano dos animais que recebem maior nível de suplementação no pós-parto. Moallem, Bruckental e Sklan (2004), verificaram aumento dos níveis de insulina plasmática em animais no pós-parto, sem aumento da taxa de glicose, quando estes foram submetidos ao aumento de $0,75 \mathrm{~g}$ de concentrado no período pré-parto. Essa evidência pode justificar a melhora observada no desenvolvimento folicular em animais no período pós-parto, inclusive em animais que receberam maior suplementação no pré-parto.

Wiltbank (1970), descreveram que animais bem alimentados antes do parto apresentaram menor intervalo ao primeiro cio do que aqueles submetidos a baixos níveis nutricionais no período pré-parto, independente do nível nutricional no pósparto. Haresign (1980), demonstrou que a condição ou o peso corporal ao parto é relativamente mais importante do que o nível de nutrição pós-parto.
Lucy, Staples e Michel (1991) verificaram que aumentando o balanço energético no período pósparto, diminuiu o número de folículos de 3 a $5 \mathrm{~mm}$ e de 6 a $9 \mathrm{~mm}$, e aumentou o número de folículos de 10 a $15 \mathrm{~mm}$, evidenciando a ação do balanço energético no desenvolvimento folicular, ovulação e aparecimento de cio. Concluíram também que o balanço energético só tem influência no início do crescimento folicular, sendo que após o início do ciclo estral, não há influência quanto ao crescimento e número de folículos.

$\mathrm{Na}$ Tabela 3, estão as correlações entre o início de atividade folicular (IAF), o início de crescimento folicular (ICF) e o escore corporal ao parto (ECP). Pode-se observar que um maior escore corporal ao parto está correlacionado a um menor tempo para início do desenvolvimento ovariano. Kim e Suh (2003), avaliaram o efeito da perda de escore corporal (moderada $=0,75$ pontos e acentuada $=$ 1,0-1,5 pontos) de vacas leiteiras no período seco, sobre o desempenho reprodutivo, e concluíram que o número de dias para o primeiro serviço foi maior para animais com perda acentuada (103 $\pm 7,8$ dias) do que animais com perda moderada ( $87 \pm 5,3$ dias). 
Tabela 3. Correlações entre o escore corporal ao parto (ECP) e desenvolvimento ovariano representado pelo início de atividade folicular (IAF) e início de crescimento folicular (ICF).

\begin{tabular}{ccc}
\hline \multirow{2}{*}{ Variáveis } & \multicolumn{2}{c}{$\boldsymbol{E P}$} \\
\cline { 2 - 3 } & $\mathrm{R}$ & $\mathrm{P}$ \\
\hline IAF & $-0,34$ & 0,0397 \\
ICF & $-0,31$ & 0,0569 \\
\hline
\end{tabular}

ECP - Escore corporal médio ao parto; IAF -Início de atividade folicular; ICF - Início de crescimento folicular. *R - Coeficiente de correlação; P-probabilidade.

Vacas com boa condição corporal no parto, são pouco afetadas pela mudança de peso no pósparto. Vacas com baixo escore corporal apresentam maiores intervalos de anestros pós-parto devido à perda de peso e este problema é exacerbado pelo baixo consumo de energia. Efeitos de condição corporal insuficiente na reprodução podem ser parcialmente superados pelo aumento de energia na alimentação pós-parto, cujo período é de alta demanda metabólica exigindo alimentos que contenham energia suficiente para o início do período pós-parto (WRIGHT; RUSSEL, 1984).

\section{Conclusões}

O aumento no nível de suplementação no período pré e no pós-parto, aumentou o número de animais que manifestaram cio e conceberam nos primeiros 120 dias pós-parto, assim como na manutenção da gestação.

Ocorreu diminuição linear no tempo para início de atividade folicular e início de crescimento folicular à medida que se aumentou o nível de suplementação no período pré e pós-parto.

O início de atividade folicular e início de crescimento folicular tiveram correlação negativa com o escore corporal ao parto.

\section{Referências}

ASSOCIATION OF OFFICIAL ANALYTICAL CHEMISTS - A.O.A.C. Official methods of analysis. 6.ed. Arlington, Virgínia: Keneth Helrich, 1990.

BRITT, J. H. Follicular development and fertility: Potential impacts of negative energy balance. In: NATIONAL REPRODUCTION SYMPOSIUM, 2., 1994, Pittsburg. Proceedings... Pittsburg: Jordam E. R., 1994. p.103-112.

BUTLER, W. R.; EVERETT, R. W.; COPPOCK, C. E. The relationships between energy balance, milk production and ovulation in postpartum Holstein cows. Journal of Dairy Science, Champaign, v. 53, n. 3, p. 742-748, 1981.

BUTLER, W. R.; SMITH, R. D. Interrelationships between energy and postpartum reproduction function in dairy cattle. Journal of Dairy Science, Champaign, v. 72, n. 3, p.767-783, 1989.

EDMONSON, A. J.; LEAN, I. J.; WEAVER, L. D.; FARVER, T.; WEBSTER, G. A body condition scoring chart for Holstein dairy cows. Journal of Dairy Science, Champaign, v. 72, p. 68-78, 1989.

GWAZDAUSKAS, F. C.; KENDRICK, K. W.; PRYOR, A. W.; BAYLEY, T. L. Impact of follicular aspiration on folliculogenesis as influenced by dietary energy and lactation. Journal of Dairy Science, Champaign, v. 83, n. 7, p. 1625-1634, 2000.

HARESIGN, W. Body condition, milk yield and reproduction in cattle. In: RECENTS ADVANCES IN ANIMAL NUTRITION, 1980, London. Proceedings... London: Butterworths,1980. p.107-122.

HOLDERBAUN, J. F.; SOLLENBERG, K. H. Canopy structure and nutritive value of limp grass pasture during mid-summer to early autumn. Agronomy Journal, Madison, v. 84, n. 1, p. 11-16, 1992. 
KIM, I. H.; SUH, G. H. Effect of the amount of body condition loss from the dry to near calving periods on the subsequent body condition change, occurrence of postpartum diseases, metabolic parameters and reproductive performance in Holstein dairy cows. Theriogenology, Stoneham, v. 60, n. 8, p. 1445-1456, 2003.

LOZANO, J. M.; ABECIA, J. A.; FORCADA, F.; Zarazaga L.; Alfaro B. Effect of under nutrition on the distribution of progesterone in the uterus of ewes during the luteal phase the estrous cicle. Theriogenology, Stoneham, v.49, n. 3, p.539-546, 1998.

LUCY, M. C. ADSA foundation Scholar Award. Reproductive loss in high-production dairy cattle: Where will it end? Journal of Dairy Science, Champaign, v. 84, n. 6, p. 1277-1293, 2001.

LUCY, M. C.; STAPLES, C. R.; MICHEL, F. M.; Thatcher, W. W. Energy balance and size and number of ovarian follicles detected by ultrasonography in early postpartum dairy cows. Journal of Dairy Science, Champaign, v. 74, n. 2, p. 473-482, 1991.

McNAMARA, S.; MURPHY, J. J.; RATH, M.; O’MARA, F. P. Effects of different transition diets on energy balance, blood metabolites and reproductive performance in dairy cows. Livestock Production Science, Amsterdam, v. 84 n. 3, p. 195-206, 2003.

MOALLEM, U.; BRUCKENTAL, I.; SKLAN, D. Effect of feeding pregnant and non-lactating dairy cows a supplement containing a high proportion of non-structural carbohydrates on post-partum production and peripartum blood metabolites. Animal Feed Science and Technology, Amsterdam v. 116, n. 3-4, p. 185-195, 2004.

NATIONAL RESEARCH COUNCIL - NRC. Nutrient requirements of dairy cattle. 7.ed. Washington, DC: National Academy Press, 2001.

OXENREIDER, S. L.; WAGNER, W. C. Effect of lactation and energy intake on postpartum ovarian activity in the cow. Journal of Animal Science, Champaign, v. 33, n. 5, p. 1026-1031, 1971.
PIERSON, R. A.; GINTHER, O. J. Ultrasonography of the bovine ovary. Theriogenology, Stoneham, v. 21, n. 3, p. 495-504, 1984.

POPE. W. F., MAURER, R. R.; STORMSHAK, F. Distribution of progesterone in the uterus, broads ligament and uterine arteries of beef cows. Anatomical Records, v. 203, n. 2, p. 245-250, 1982.

ROCHE, J. F.; MACKEY, D.; DISKIN, M. D. Reproductive management of postpartum cows. Animal Reproduction Science, Amsterdam, v. 60-61, n. 2, p.703712, 2000.

SANTOS, J. E. P.; AMSTALDEN, M. Effects of nutrition on bovine reproduction. Arquivos da faculdade de veterinária UFRGS, Porto Alegre, v. 26, n. 1, p. 1989, 1998.

SNIJDERS, S. E.; DILLON, V.; O'CALLAGHAN, D.; BOLAND, M. P. Effect of genetics merit, milk yield, body condition and lactation nuymber on in vitro oocyte development in dairy cows. Theriogenology, Stoneham, v. 53, n. 4, p. $981-989,2000$.

SPICER, L. J.; ENRIGHT, W. J. Concentration of insuline-like growth factor I and esteroids in follicular fluid of preovulatory bovine ovarian follicles: efects of daily injection of growth hormone-resleasing hormone. Journal of Animal Science, Champaign, v. 69, n. 3, p.1133-1139, 1991.

THATCHER, W. W.; WILCOX, C. J. Postpartum estrus as an indicator of reproductive status in the dairy cow. Journal of Dairy Science, Champaign, v. 56, n. 5, p. 608610, 1972.

WILTBANK, J. N. Research needs in beef cattle reproduction. Journal of Animal Science, Champaign, v. 31, n. 4, p. 755-762, 1970.

WRIGHT, I. A.; RUSSEL, A. F. J. Partition of fat, body composition score in mature cows. Animal Production, Bletchley, v. 38, n. 1, p. 23-32, 1984. 